\title{
Some records of Limoniidae and Pediciidae (Diptera) from Portugal and Spain
}

Jaroslav Starý

Some records of Limoniidae and Pediciidae (Diptera) from Portugal and Spain. - Acta Mus. Siles. Sci. Natur., 63: 83-95, 2014.

Abstract: Records of species of the families Limoniidae and Pediciidae are listed from Portugal and Spain within the political borders of these countries, i.e. including Azores, Madeira and Balearic Islands, Canary Islands, respectively. A total of 56 species of Limoniidae and two species of Pediciidae are registered. Of these 43 species of Limoniidae and one species of Pediciidae are new to Portugal and/or Spain, or some of their overseas areas.

Key words: Diptera, Limoniidae, Pediciidae, distribution, Portugal, Spain.

\section{Introduction}

Although attention has long been paid to the fauna of Limoniidae and Pediciidae of the Iberian Peninsula and especially that of the Macaronesian archipelagos, the knowledge of this fauna is by far not sufficient, mainly for the continental Portugal and Balearic Islands. Recently, an extensive material accumulated through collections of my colleagues and myself, which represents some important faunal additions. With the exception of Andorra, which is not covered by material at my disposal, the present paper encompasses the same territory as does the recently published checklist (Eiroa \& Báez 2002). This checklist may thus serve as a basis for enumeration of species. Since that time, however, further papers were published, with records and descriptions of the Portuguese and Spanish Limoniidae and Pediciidae, such as Carles-Tolrá, Calvo \& Zabalegui (2006), Carles-Tolrá \& Lencina (2010), Carles-Tolrá \& Ventura (2009), Starý (2006a, 2006b, 2008, 2009a, 2009b, 2011, 2012), and Starý \& Oosterbroek (2008). Principally, records of all species available to me from the territory and hitherto unpublished are listed here, including those already recorded from a given area, except for species of uncertain identity and those new to science. Altogether 56 species of Limoniidae and two species of Pediciidae are listed. Of these 43 species of Limoniidae and one species of Pediciidae are new to Portugal and/or Spain, or some of their overseas areas.

\section{Material and methods}

Taxa from family to species are arranged here in alphabetical order. With each species entry, the examined material is listed according to the two countries, Portugal and Spain (continental), followed by material from overseas areas, viz. Azores, Madeira and Balearic Islands, Canary Islands, respectively. Within each territorial unit, localities for a species are roughly arranged from north to south and west to east. All specimens listed here are dried, pinned or glued onto points, and deposited in the collection of the author. The specimens were mostly collected by sweeping vegetation with a net or individual netting. The male and female terminalia, if necessary, were prepared by boiling in a solution of $10 \% \mathrm{KOH}$ and preserved in glycerine in a sealed plastic tube pinned with the appropriate specimen. Distribution as it is given here for individual species is based on Oosterbroek (2014). For more detailed distribution of the Macaronesian Limoniidae lists of species in recent publications may be sometimes consulted, such as Báez \& García (2004), Eiroa (2008), and Vieira, Diaz \& Báez (2010). 


\section{LIMONIIDAE: CHIONEINAE}

\section{Baeoura ebenina Starý, 1981}

\section{Material examined:}

Portugal: Algarve: Tavira env., Portela (Ribeiro), Odeleite river (150 m), 1.iv.2009, 1 q; Tavira env., Casa Queimada, $1 \mathrm{~km} \mathrm{~N}$, brook (124 m), 31.iii.2009, 8 §,, 5 o; Tavira env., Currais Boieiros, river (27 m), 31.iii.2009, 1 §’; Azinhal env., Barreiros, 1 km S, Beliche river (75 m), 2.iv.2009, 1 ㅇ, 4.iv.2009, 3 ऽ, 3 우 (all J. Starý leg.).

Spain: Andalucía: Coin, Rio Seco, 7.iv.2005, 1 † (J. Starý leg.)

Distribution: Only known from Spain. First records since original description; first records for Portugal.

\section{Cheilotrichia (Empeda) cinerascens (Meigen, 1804)}

Material examined:

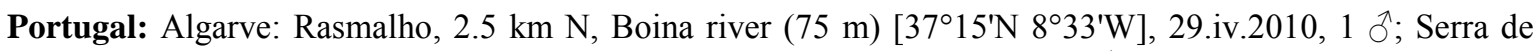

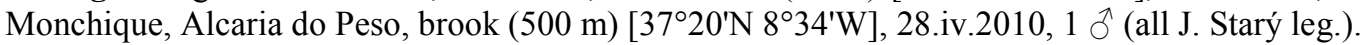

Spain: Andalucía: Cortijo Los Pinos (nr. Alozaina), road bridge, 3.iv.2005, 1 ô, 1 o; Coin, Rio Seco, 30.iii.2005, 2 ô, 4.iv.2005, 1 ô, 7.iv.2005, 2 ô, 2 क; Fuengirola, Rio de Fuengirola, 29.iii.2005, 3 సं; Cártama, Rio Guadalhorce, 31.iii.2005, 1 ○; Benalmádena, 28.iii.2005, 1 đ̊ (all J. Starý leg.).

Distribution: Europe (unrecorded from Portugal); Georgia, Turkey, Cyprus, Israel, Iran. First records for Portugal.

\section{Cheilotrichia (Empeda) fuscohalterata (Strobl, 1906)}

Material examined:

Spain: Andalucía: Alhaurin el Grande, 5 km E, 30.iii.2005, 1 §’; Cártama, Rio Guadalhorce, 31.iii.2005, $1 \widehat{\sigma}$ (all J. Starý leg.).

Distribution: Italy (Sardinia), Portugal, Spain.

\section{Erioconopa diuturna (Walker, 1848)}

Material examined:

Portugal: Algarve: Vila do Bispo, 4 km NE, Lagoa Funda, marsh (126 m) [3707'N 854'W], 30.iv.2010, 5 ô, 3.v.2010, 3 ô (J. Starý leg.).

Spain: Balearic Is: Mallorca: Polença, 4.8 km SE, brooks (14 m) [3951'11"N 303'38"E], 3.v.2013, 4 ð̊ (J. Starý leg.).

Distribution: Europe (unrecorded from Portugal); Morocco; Turkey. First record for Portugal and Spain (Balearic Is).

\section{Erioptera (Erioptera) fuscipennis Meigen, 1818}

\section{Material examined:}

Portugal: Algarve: Serra de Monchique, Foia Mt., springs and marshes (780-870 m) [37¹9'N 8³6'W],

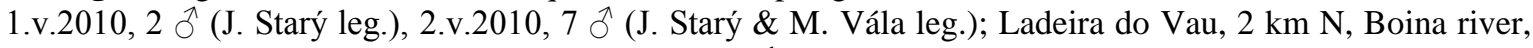

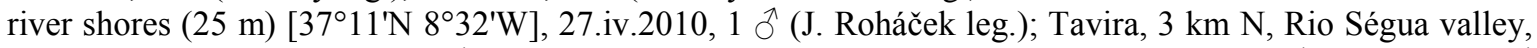
drainages (24 m), 1.iv.2009, 5 §̊; Monte Gordo, 1 km N, saltmarsh (13 m), 30.iii.2009, $1 \hat{\jmath}, 4.1 \mathrm{v} .2009,1 \hat{\jmath}$ (all J. Starý leg.).

Spain: Andalucía: Pizzara, Rio Guadalhorce, 3.iv.2005, 8 §; Casapalma, confl. Rio Grande and Rio

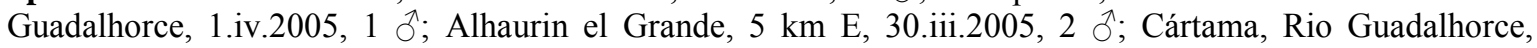

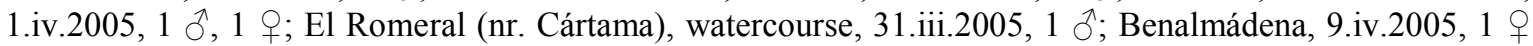
(all J. Starý leg.). Balearic Is: Mallorca: S’Albufera National Park, “Cami des Polls”, saltmarsh and canal (0-

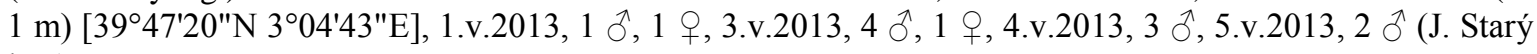
leg.).

Distribution: Europe (unrecorded from Portugal); Morocco, Algeria; Azerbaijan, Iran. First records for Portugal and Spain (Balearic Is).

\section{Gonomyia (Gonomyia) sicula Lackschewitz, 1940}

Material examined:

Spain: Andalucía: 15 km NM Jimena de la Frontera, cork-oak forest (400 m), 17.iv.1994, 1 đ̊ 
(P. Oosterbroek \& C. Hartveld leg.); Grazalema nr. Ronda (1000 m), 16.-17.v.1979, 1 ふ̊; Loja env., 15.v.1979, 1 đ (all J. Roháček leg.).

Distribution: Italy (incl. Sicily), Spain; Tunisia; Turkey.

\section{Hoplolabis (Parilisia) obtusiapex (Savchenko, 1982)}

Material examined:

Spain: Andalucía: Casapalma, confl. Rio Grande and Rio Guadalhorce, 1.iv.2005, 3 đ̊; Cártama, Rio

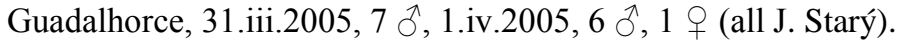

Distribution: Belarus, Bulgaria, Czech Republic, Greece, Macedonia, Moldovia, Romania, Slovakia, Spain, Ukraine; Morocco, Algeria.

\section{Hoplolabis (Parilisia) sororcula (Lackschewitz, 1940)}

Material examined:

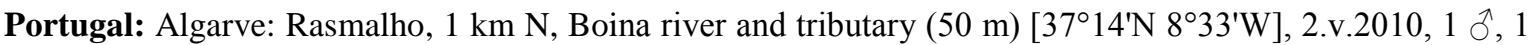
+ (J. Starý leg.).

Spain: Andalucía: Cortijo Los Pinos nr. Alozaina, 3.iv.2005, 1 §; Coin, Rio Seco, 4.iv.2005, 1 đ, 7.iv.2005,

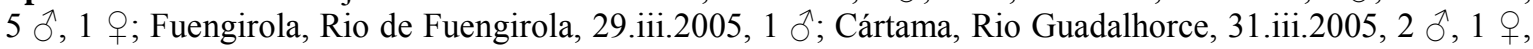
1.iv.2005, 2 ๙ (all J. Starý leg.).

Distribution: France, Germany, Italy (incl. Sicily), Romania, Slovakia, Spain, Switzerland, Ukraine; Morocco. First record for Portugal.

\section{Idiocera (Idiocera) pulchripennis (Loew, 1856)}

Material examined:

Spain: Andalucía: Cártama, Rio Guadalhorce, 1.iv.2005, 1 q (J. Starý leg.). Balearic Is: Mallorca:

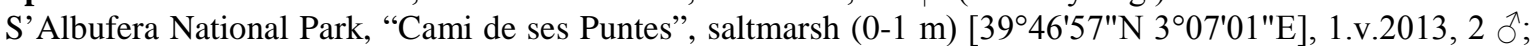
S’Albufera National Park, “Cami des Polls”, saltmarsh and canal (0-1 m) [3947'20"N 304'43"E], 4.v.2013, 1 ठै (all J. Starý leg.).

Distribution: Europe (unrecorded from Portugal and continental Spain), except for northern countries; Morocco, Algeria, Egypt; Transcaucasia, Turkey, Cyprus, Israel, Iran; Central Asia. First records for continental Spain and Balearic Is.

\section{Idiocera (Idiocera) sziladyi (Lackschewitz, 1940)}

Material examined:

Portugal: Algarve: Tavira env., Portela (Ribeiro), Odeleite river (150 m), 1.iv.2009, 2 ふૈ; Tavira env., Currais Boieiros, river $(27 \mathrm{~m}), 31 . i i i .2009,1$ ડ̊; Azinhal env., Barreiros, $1 \mathrm{~km} \mathrm{~S}$, Beliche river (75 m), 2.iv.2009, 1 ㅇ (all J. Starý leg.).

Spain: Andalucía: Zalea (nr. Pizzara), Arroyo de Casarabonela, 3.iv.2005, 2 ô, 1 o; Pizzara, Rio

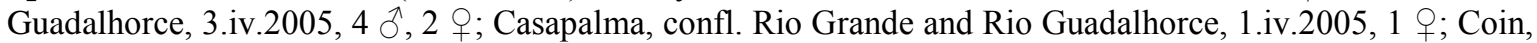

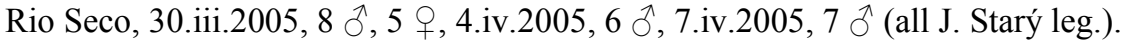

Distribution: Europe (unrecorded from Portugal), except for northern countries; Algeria, Egypt; Yemen. First records for Portugal.

\section{Ilisia maculata (Meigen, 1804)}

Material examined:

Spain: Andalucía: Benalmádena, 9.iv.2005, 1 đ̋ (J. Starý leg.).

Distribution: Europe (unrecorded from Portugal); Transcaucasia, Turkey, Iran.

\section{Molophilus (Molophilus) baezi Theowald, 1981}

Material examined:

Portugal: Madeira: Ribeira da Janela, 3 km S, sweeping along levadas in laurel forest (850 m), 10.ix.2003,

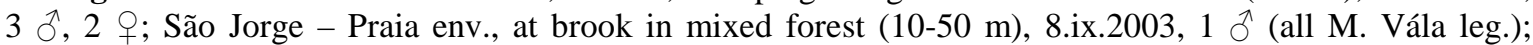
Feiteira do Nuno nr. Santana, at brook in mixed forest (600 m), 8.ix.2003, 1 đิ (J. Roháček leg.); Rabaçal [32.46N 17.08W], 3.iii.2011, 1 ふో; Funchal - Corujeira, $1.5 \mathrm{~km} \mathrm{~N}$, at brook in mixed forest $(600 \mathrm{~m})$, 13.ix.2003, 1 ○े (all M. Vála leg.).

Distribution: Only known from Portugal (Madeira) and Spain (Canary Is). 


\section{Molophilus (Molophilus) griseus (Meigen, 1804)}

Material examined:

Spain: Galicia: Lugo env., $10 \mathrm{~km} \mathrm{E} \mathrm{Foz,} \mathrm{meadows} \mathrm{in} \mathrm{pine} \mathrm{forest}(20 \mathrm{~m}), 18 . v .1994,1$ ( (P. Oosterbroek \& C. Hartveld leg.).

Distribution: Europe (unrecorded from Portugal and questionable for Spain); Algeria; Georgia, Azerbaijan, Turkey, Israel. After M. (M.) testaceus (previously a "variety" of griseus) was raised to species rank (Starý 2011), this is an important confirmation of the occurrence of M. (M.) griseus in Spain.

\section{Molophilus (Molophilus) pleuralis de Meijere, 1920}

Material examined:

Spain: Balearic Is: Mallorca: S'Albufera National Park, "Cami des Polls", saltmarsh and canal (0-1 m) [3947'20"N 304'43"E], 4.v.2013, 2 § , 1 q (J. Starý leg.).

Distribution: Europe (unrecorded from Portugal and Spain); Transcaucasia, Turkey, Cyprus, Israel, Iran; Central Asia; as far east as Far East of Russia. First record for Spain (Balearic Is).

\section{Molophilus (Molophilus) testaceus Lackschewitz, 1940}

Material examined:

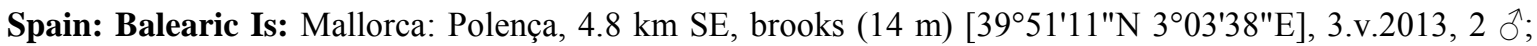
S'Albufera National Park, "Cami des Polls", saltmarsh and canal (0-1 m) [3947'20"N 3०04'43"E] 3.v.2013, 3 ภิ, 4.v.2013, 7 ठ̂, 5.v.2013, 1 ڤ̂ (all J. Starý leg.).

Distribution: Only known from Portugal and Spain. First records for Spain (Balearic Is).

\section{Phyllolabis hemmingseni Nielsen, 1959}

Material examined:

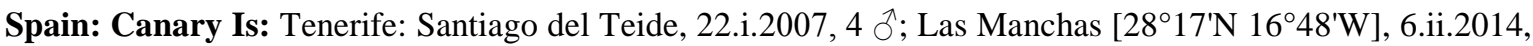

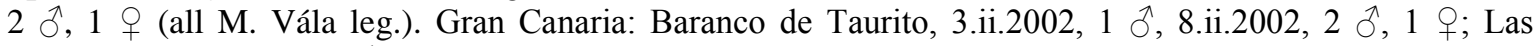
Lagunetas, 17.ii.2004, 2 đ, 1 ๆ (all M. Vála leg.).

Distribution: Only known from Spain (Canary Is).

\section{Rhabdomastix (Rhabdomastix) eugeni Starý, 2004}

Material examined:

Spain: Andalucía: Casapalma, confl. Rio Grande and Rio Guadalhorce, 1.iv.2005, 16 ${ }^{\lambda}, 4$ 우 Coin, Rio

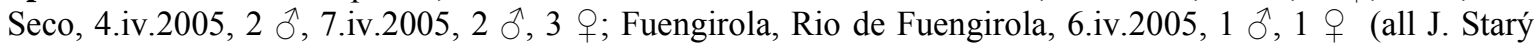
leg.).

Distribution: Europe (unrecorded from Portugal and Spain), except for northern countries; Armenia. First records for Spain.

\section{Scleroprocta oosterbroeki Starý, 2008}

Material examined:

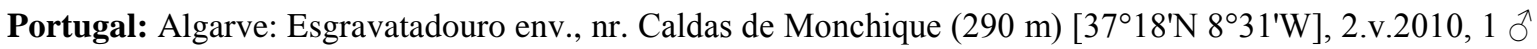
(J. Starý leg.).

Distribution: Only known from Spain. First record since original description; first record for Portugal.

\section{Symplecta (Psiloconopa) stictica (Meigen, 1818)}

Material examined:

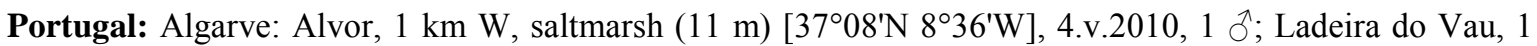
$\mathrm{km}$ N, Boina river, saltmarsh $(24 \mathrm{~m})\left[37^{\circ} 10^{\prime} \mathrm{N} 8^{\circ} 32^{\prime} \mathrm{W}\right]$, 27.iv.2010, $2{ }^{\circ}$; Castro Marim, $0.3 \mathrm{~km} \mathrm{~S}$, saltmarsh

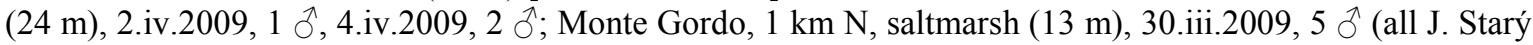
leg.).

Spain: Balearic Is: Mallorca: S'Albufera National Park, "Cami de ses Puntes", saltmarsh $(0-1 \mathrm{~m})$

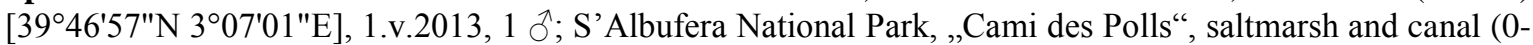

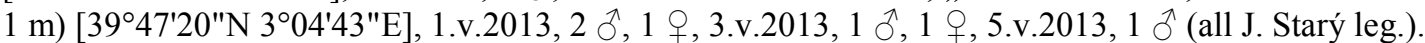

Distribution: Europe (unrecorded from Portugal); Transcaucasia, Turkey, Israel, Iran; Turkmenistan, Afghanistan. First records for Portugal. 


\section{Symplecta (Symplecta) grata Loew, 1873}

Material examined:

Spain: Balearic Is: Mallorca: S’Albufera National Park, “Cami des Polls”, saltmarsh and canal (0-1 m)

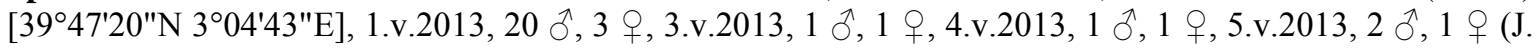
Starý leg.).

Distribution: Nearctic (USA: Utah); Austria, Czech Republic, ?Hungary, Italy (Sicily), Romania, Slovakia, Spain, Ukraine; Algeria; Central Asia, West Siberia. First records for Spain (Balearic Is).

\section{Symplecta (Symplecta) hybrida (Meigen, 1804)}

Material examined:

Portugal: Azores: São Miguel I.: Sete Cidades env., 31.viii.2006, 1 đ, 1 q (M. Vála leg.); Lombadas, shores of Ribeira Grande, 5.ix.2006, 3 ภ, 1 q; Ribeira Grande, 2 km SE, 5.ix.2006, 8 ๙, 2 q (J. Roháček \& M. Vála leg.); Lagoa do Fogo, brook, 7.ix.2006, 2 \& (M. Vála).

Spain: Andalucía: Casapalma, confl. Rio Grande and Rio Guadalhorce, 1.iv.2005, 1 \%; Coin, Rio Seco, 7.iv.2005, 1 đ̃ (all J. Starý leg.).

Distribution: Nearctic (Canada, USA, Greenland); widespread in Palaearctic (unrecorded from continental Portugal), including North Africa, Central Asia, Mongolia, as far east as North Korea and Japan; Oriental (India, Nepal, Pakistan).

\section{Symplecta (Trimicra) pilipes (Fabricius, 1787)}

Material examined:

Portugal: Algarve: Vila do Bispo, 4 km NE, Lagoa Funda, marsh (126 m) [3707'N 854'W], 30.iv.2010, 1 $\delta^{\top}$; Alvor, Luna Alvor Village hotel (at light) $\left[37^{\circ} 08^{\prime} \mathrm{N} 8^{\circ} 35^{\prime} \mathrm{W}\right], 26 . i v .2010,1 \delta^{\lambda}, 1$ of; Alvor, $1 \mathrm{~km} \mathrm{~W}$,

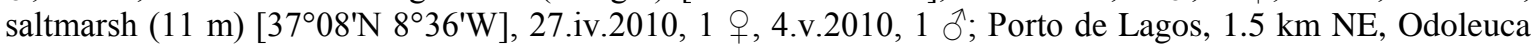
river $(24 \mathrm{~m})\left[37^{\circ} 12^{\prime} \mathrm{N} 8^{\circ} 31^{\prime} \mathrm{W}\right], 28 . i v .2010,1{ }^{\top}$; Porto de Lagos, $1.5 \mathrm{~km} \mathrm{NW}$, Boina river $(25 \mathrm{~m})\left[37^{\circ} 12^{\prime} \mathrm{N}\right.$ $8^{\circ} 32^{\prime} \mathrm{W}$ ], 29.iv.2010, $1 \mathrm{o}^{\top}$; Ladeira do Vau, $1 \mathrm{~km} \mathrm{~N}$ (Boina river), saltmarsh $(24 \mathrm{~m})\left[37^{\circ} 10^{\prime} \mathrm{N} 8^{\circ} 32^{\prime} \mathrm{W}\right]$, 27.iv.2010, 2 đ๋ (all J. Starý leg.); Monte Gordo, seashore (2 m), 30.iii.2009, 1 ๆ (M. Vála leg.).

Spain: Andalucía: Casapalma, confl. Rio Grande and Rio Guadalhorce, 1.iv.2005, 1 §̊; Alhaurin el Grande,

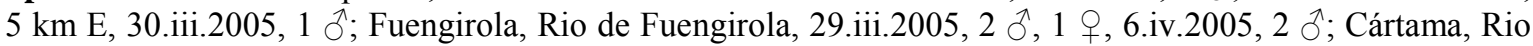
Guadalhorce, 31.iii.2005, 1 đో; Benalmádena, 28.iii.2005, 1 đ̂ (all J. Starý leg.). Balearic Is: Mallorca: S’Albufera National Park, “Cami des Polls”, saltmarsh and canal (0-1 m) [3947'20"N 304'43"E], 3.v.2013,

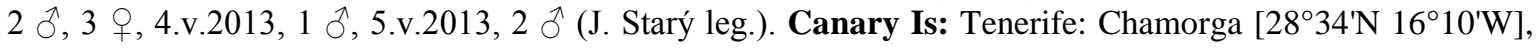
8.ii.2014, 1 q (M. Vála leg.).

Distribution: Cosmopolitan (unrecorded from continental Portugal). First records for Portugal and Spain (Balearic Is: Mallorca)..

\section{Tasiocera (Dasymolophilus) minima Mendl, 1974}

Material examined:

Spain: Balearic Is: Mallorca: Fangar nr. Campanet, Torrent Massana, nr. brook (65 m) [3948'37"N 2॰58'28"E], 28.iv.2013, 2 ふૈ, 2.v.2013, 6 ふ (J. Starý leg.).

Distribution: Only known from Italy (incl. Sicily). First records for Spain (Balearic Is).

\section{LIMONIIDAE: LIMNOPHILINAE}

\section{Austrolimnophila (Austrolimnophila) analis (Santos Abreu, 1923)}

\section{Material examined:}

Portugal: Madeira: Ribeira da Janela (850 m), 10.ix.2003, 2 ð, 1 q (M. Vála leg.); Chão de Cancela nr. Seixal (500 m), 3.ix.2003, 5 đ̄, 2 q (J. Roháček \& M. Vála leg.); Feiteira do Nuno nr. Santana (600 m), 8.ix.2003, 6 đ̃, 1 km SW (700 m), 9.ix.2003, 4 ô, 1 o (J. Roháček leg.); Rabaçal env. (950 m), 5.ix.2003, 1

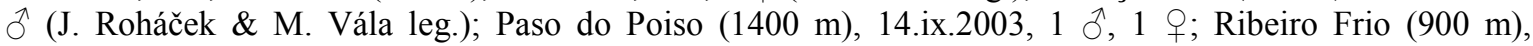

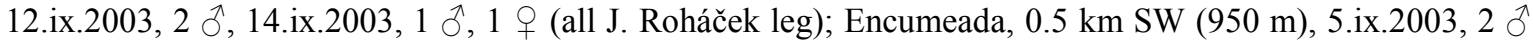
(J. Roháček \& M. Vála leg.).

Spain: Canary Is: Tenerife: Vueltas de Taganana, laurel forest, 4.v.1999, 2 ふో; Anaga Mts, Cruz del Carmen, 1 km NE, 8.v.1999, 2 đ̊; Agua Garcia nr. Tacoronte, laurel forest, 3.v.1999, 2 đ̊ (all J. Roháček leg.).

Distribution: Only known from Spain (Canary Is: Gomera, La Palma). First records for Portugal (Madeira) and Spain (Canary Is: Tenerife). 


\section{Austrolimnophila (Austrolimnophila) latistyla Starý, 1977}

\section{Material examined:}

Portugal: Viana do Castelo: Serra do Peneda, Soajo - Lindosa, 5.vi.1998, 1 đ̊ (A. Čelechovský leg.). Algarve: Serra de Monchique, Alcaria do Peso, brooks (450-500 m), 3.iv.2009, 5 ô, 4 q, brook (500 m)

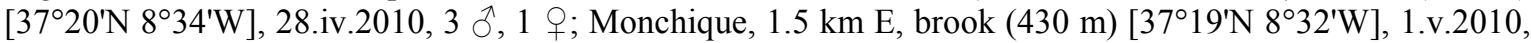

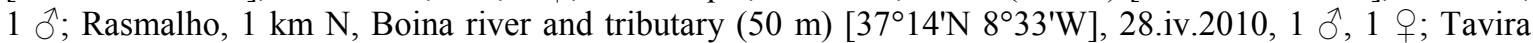
env., Cachopo, 5 km SE, artificial lake (210 m), 1.iv.2009, 2 §, 1 q; Tavira env., Portela (Ribeiro), Odeleite river (150 m), 1.iv.2009, 1 ; Azinhal env., Barreiros, $1 \mathrm{~km} \mathrm{~S}$, Beliche river (75 m), 2.iv.2009, 3 §, 2 ㅇ (all J. Starý leg.).

Spain: Andalucía: Coin, Rio Seco, 7.iv.2005, 1 § ; Fuengirola, Rio de Fuengirola, 29.iii.2005, $2 \widehat{\delta}$ (all J. Starý). Balearic Is: Mallorca: S’Albufera National Park, "Cami des Polls”, saltmarsh and canal (0-1 m) [39॰47'20"N 304'43"E], 1.v.2013, 1 ㅇ, 3.v.2013, 1 ㅇ, 4.v.2013, 1 ô, 1 ๆ (J. Starý leg.)

Distribution: Croatia, France (incl. Corsica), Greece (Evia, Crete), Italy (incl. Lipari Is), Spain. First records for Portugal and Spain (Balearic Is).

\section{Euphylidorea aperta (Verrall, 1887)}

Material examined:

Portugal: Algarve: Serra de Monchique, Foia Mt., springs and marshes (780-870 m) [37019'N 8³6'W], 2.v.2010, 3 ふ̄ (J. Starý leg.).

Distribution: Europe (unrecorded from Portugal and Spain), except for northern countries. First record for Portugal.

\section{Hexatoma (Hexatoma) obscura (Meigen, 1818)}

\section{Material examined:}

Portugal: Algarve: Rasmalho, $1 \mathrm{~km}$ N, Boina river and tributary $(50 \mathrm{~m})$ [37 $\left.7^{\circ} 14^{\prime} \mathrm{N} 8^{\circ} 33^{\prime} \mathrm{W}\right]$, 28.iv.2010, $2 \mathrm{\delta}^{\top}$; Porto de Lagos, $1.5 \mathrm{~km} \mathrm{NE}$, Odolouca river $(24 \mathrm{~m})\left[37^{\circ} 12^{\prime} \mathrm{N} 8^{\circ} 31^{\prime} \mathrm{W}\right]$, 28.iv.2010, 1 ${ }^{\lambda}, 1.5 \mathrm{~km}$ NW, Boina

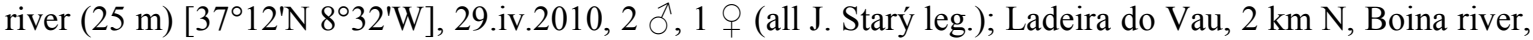
river shores $(25 \mathrm{~m})\left[37^{\circ} 11^{\prime} \mathrm{N} 8^{\circ} 32^{\prime} \mathrm{W}\right], 27 . i v .2010,1$ đ (J. Roháček leg.); Tavira env., Portela (Ribeiro),

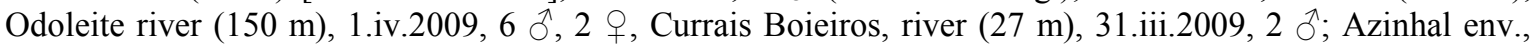
Barreiros, 1 km S, Beliche river (75 m), 2 iv.2009, 2 ऽ, 3 ㅇ, 4.iv.2009, 1 § (all J. Starý leg.).

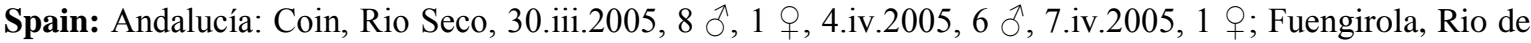
Fuengirola, 6.iv.2005, 4 ๙ิ (all J. Starý).

Distribution: Portugal, Spain; Mongolia.

\section{Paradelphomyia (Oxyrhiza) senilis (Haliday, 1833)}

Material examined:

Portugal: Algarve: Esgravatadouro env., nr. Caldas de Monchique (290 m) [37018'N 8³1'W], 2.v.2010, 1 ô, 2 q (J. Starý leg.).

Distribution: Europe (unrecorded from Portugal); Azerbaijan, Turkey; Kirgiziya. First record for Portugal.

\section{Phylidorea (Phylidorea) ferruginea (Meigen, 1818)}

Material examined:

Portugal: Algarve: Vila do Bispo, 4 km NE, Lagoa Funda, marsh (126 m) [3707'N 854'W], 30.iv.2010, 1 ô, 3.v.2010, 1 đ̃ (J. Starý leg.).

Spain: Balearic Is: Mallorca: S’Albufera National Park, “Cami des Polls”, saltmarsh and canal (0-1 m) [3947'20"N 304'43"E], 3.v.2013, 1 đ̃, 2 ㅇ, 4.v.2013 1 ㅇ, 5.v.2013, 1 ô (J. Starý leg.).

Distribution: Europe (unrecorded from Portugal); Azerbaijan, Turkey, Israel; West Siberia, Central Asia, Mongolia. First records for Portugal and Spain (Balearic Is).

\section{Pseudolimnophila (Pseudolimnophila) ebullata Starý, 1982}

Material examined:

Portugal: Algarve: Serra de Monchique, Caldas de Monchique, brooks (230 m), 3.iv.2009, 4 đ (J. Roháček

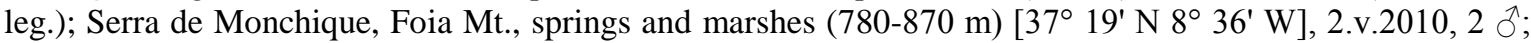
Serra de Monchique, Alcaria do Peso, brook (500 m) [37²0' N 8 34' W], 28.iv.2010, 1 ô (all J. Starý leg.). Spain: Andalucía: Alhaurin el Grande, 5 km E, 30.iii.2005, 1 o; Benalmádena, 9.iv.2005, $3 \hat{\jmath}, 1$ ๆ (all J. Starý leg.).

Distribution: Italy (Sicily), Spain. First records for Portugal. 


\section{LIMONIIDAE: LIMONIINAE}

\section{Achyrolimonia decemmaculata (Loew, 1873)}

Material examined:

Portugal: Algarve: Serra de Monchique, Alcaria do Peso, brooks (450-500 m), 3.iv.2009, 1 ๙ , brook (500 m) $\left[37^{\circ} 20^{\prime} \mathrm{N} 8^{\circ} 34^{\prime} \mathrm{W}\right], 28 . i v .2010,1{ }^{\top}$; Monchique, $1.5 \mathrm{~km}$ E, brook (430 m) [37²19'N 8³2'W], 1.v.2010, 2 ऽิ (all J. Starý leg.).

Distribution: Europe (unrecorded from Portugal and Spain); Transcaucausia, Iran. First records for Portugal.

\section{Atypophthalmus (Atypophthalmus) quinquevittatus (Santos Abreu, 1923)}

Material examined:

Portugal: Madeira: Maloeira nr. Raposeira do Lugarinho (650 m), 10.ix.2003, 1 đ (J. Roháček leg.); Rabaçal env., (950 m), 5.ix.2003, 1 §̋ (J. Roháček \& M. Vála leg.); Funchal - Corujeira, 1 km N, 11.ix.2003, 1 đ̆ (J. Roháček leg.).

Distribution: Only known from Spain (Canary Is). First records for Portugal (Madeira).

\section{Dicranomyia (Dicranomyia) chorea (Meigen, 1818)}

Material examined:

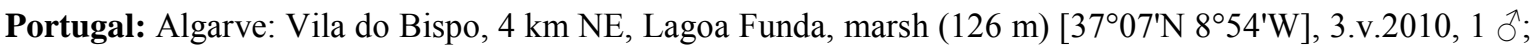
Serra de Monchique, Foia Mt., springs and marshes (780-870 m) [37²19'N $\left.8^{\circ} 36^{\prime} \mathrm{W}\right]$, 1.v.2010, 1 ;

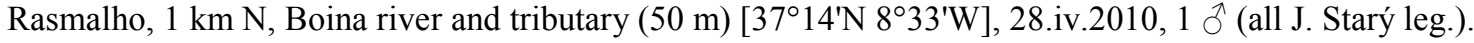

Spain: Balearic Is: Mallorca: Fangar nr. Campanet, Torrent Massana (nr. brook) (65 m) [3948'37"N $2^{\circ} 58^{\prime} 26^{\prime \prime E}$ ], 2.v.2013, 3 ; Binibona nr. Selva (nr. brook) (115 m) [3947'05"N 2॰56'32"E], 5.v.2013, 1 q (all J. Starý leg.).

Distribution: Nearctic (Canada); Europe (unrecorded from continental Portugal); Morocco; Transcaucasia, Turkey, Cyprus, Israel, Iran. First records for Portugal and Spain (Balearic Is).

\section{Dicranomyia (Dicranomyia) longicollis (Macquart, 1846)}

Material examined:

Portugal: Algarve: Tavira env., Currais Boieiros, river $(27 \mathrm{~m}), 31 . i i i .2009,5$ ๙, 2 q; Azinhal env., Barreiros, 1 km S, Beliche river (75 m), 2.iv.2009, 2 §, 2 q (all J. Starý leg.).

Spain: Andalucía: El Burgo, Rio Turón, 5.iv.2005, 2 ơ; Sierra de Ajpujata, between Ojén and Monda,

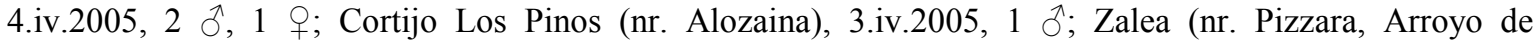

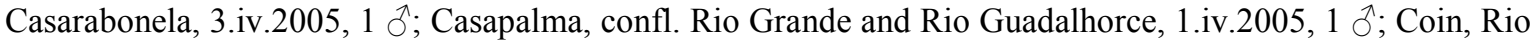
Seco, , 30.iii.2005, 5 ô, 1 o ; Coin, Rio Perelias, 3.iv.2005, 1 q; Alhaurin el Grande, 5 km E, 30.iii.2005, 11

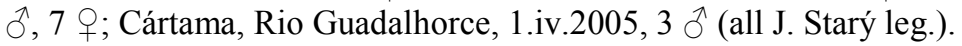

Distribution: Spain; Morocco, Algeria. First records for Portugal.

\section{Dicranomyia (Dicranomyia) longipennis (Schummel, 1829)}

Material examined:

Portugal: Algarve: Vila do Bispo, 4 km NE, Lagoa Funda, marsh (126 m) [3707'N 854'W], 30.iv.2010, 2

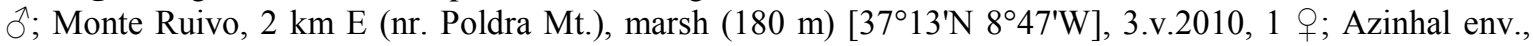
Barreiros, 1 km S, Beliche river (75 m), 4.iv.2009, 1 § (all J. Starý leg.).

Distribution: Nearctic (Canada, USA); widespread over Palaearctic (unrecorded from Portugal), including Central Asia and Mongolia, as far east as Far East of Russia and Japan; Oriental (India). First records for Portugal.

\section{Dicranomyia (Dicranomyia) maderensis (Wollaston, 1858)}

Material examined:

Portugal: Madeira: Ribeira da Janela, 3 km S, laurel forest ( $850 \mathrm{~m}), 10 . i x .2003,2$ (M. Vála leg.); Paso do

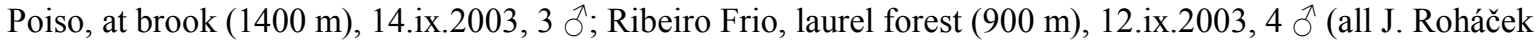
leg.); Encumeada, $0.5 \mathrm{~km} \mathrm{SW}$, spring areas (950 m), 5.ix.2003, 1 q; Rabaçal env., laurel forest (950 m), 5.ix.2003, 2 \& (all J. Roháček \& M. Vála leg.); Terreiro da Luta nr. Funchal, along levada (800 m), 14.ix.2003, 1 §ं; Funchal - Corujeira, 1.5 km N, at brook (600 m), 13.ix.2003, 1 o, 1 ○ (all M. Vála leg.);

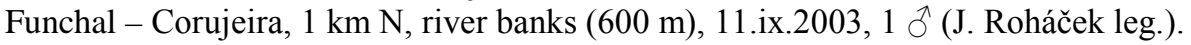

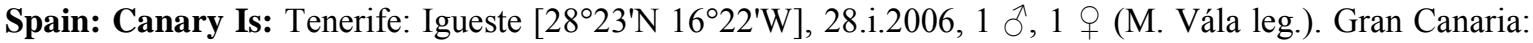
Las Lagunetas, 17.ii.2004, 6 §ึ, 1 q (M. Vála leg.). 
Distribution: Only known from Portugal (Madeira). First records for Spain (Canary Is).

\section{Dicranomyia (Dicranomyia) modesta (Meigen, 1818)}

Material examined:

Portugal: Algarve: Carrapateira, Carrapateira river (24 m) [37²11'N 853'W], 30.iv.2010, $1 \delta^{`}$; Monte Ruivo,

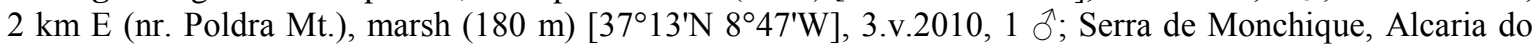

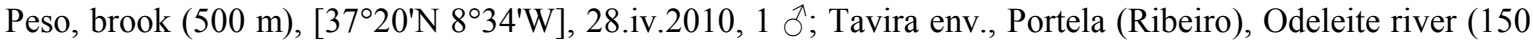
m), 1.iv.2009, 1 §ं; Tavira env., Currais Boieiros, river (27 m), 31.iii.2009, 1 ô (all J. Starý leg.).

Spain: Andalucía: Zalea (nr. Pizzara), Arroyo de Casarabonela, 3.iv.2005, 1 §̊; Cártama, Rio Guadalhorce, 31.iii.2005, 1 §๋; Benalmádena, 28.iii.2005, 1 § (all J. Starý leg.). Canary Is: Tenerife: Puerto de la Cruz, 21.i.2007, 1 §̂, 1 \& (M. Vála leg.).

Distribution: Nearctic (Canada, USA, Greenland); widespread over Palaearctic (unrecorded from Portugal and overseas Spain), including Central Asia and Mongolia, as far east as Far East of Russia and Japan. First records for Portugal and Spain (Canary Is).

\section{Dicranomyia (Dicranomyia) novemmaculata (Strobl, 1906)}

Material examined:

Portugal: Algarve: Serra de Monchique, Alcaria do Peso, brooks (450-500 m), 3.iv.2009, 1 \&; Tavira env., Portela (Ribeiro), Odeteite river (150 m), 1.iv.2009, 1 §̃, 1 q; Azinhal env., Barreiros, 1 km S, Beliche river

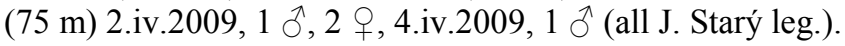

Distribution: Gibraltar, Spain; Algeria. First records for Portugal.

\section{Dicranomyia (Dicranomyia) patricia Starý, 1982}

Material examined:

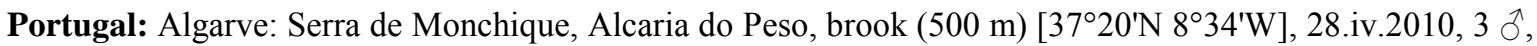

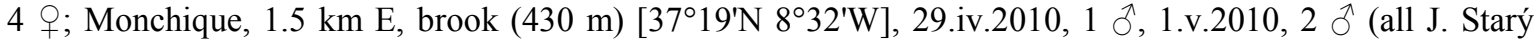
leg.).

Distribution: ?Bulgaria; Tunisia. First records since original description; first records for Portugal.

\section{Dicranomyia (Dicranomyia) sera (Walker, 1848)}

\section{Material examined:}

Spain (Balearic Is): Mallorca: S'Albufera National Park, "Cami de ses Puntes", saltmarsh (0-1 m)

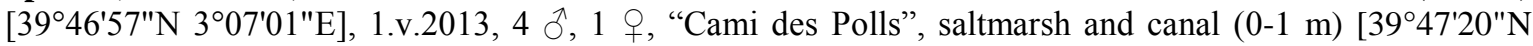
$\left.3^{\circ} 04^{\prime} 43^{\prime \prime E}\right], 1 . v .2013,11$ §े, 2 ○ (J. Starý leg.).

Distribution: Nearctic (Canada, USA); Europe (unrecorded from Portugal and Spain); Turkey; Central Asia, Mongolia; as far east as Far East of Russia. First records for Spain (Balearic Is).

\section{Dicranomyia (Dicranomyia) vicina (Macquart, 1839)}

Material examined:

Portugal: Azores: São Miguel I.: Santo Anttonio, Da Atalhada reserve, wet leafy forest, 6.ix.2006, $1 \delta$ (J. Roháček \& M. Vála leg.). Madeira: Rabaçal env., laurel forest (950 m), 5.ix.2003, 1 ㅇ (J. Roháček \& M.

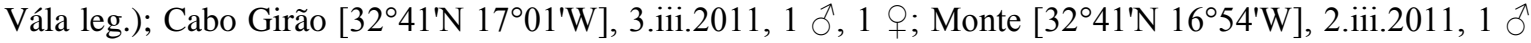
(all M. Vála leg.); Funchal - Penteada, riverside vegetation (150 m), 6.ix.2003, 1 o; Funchal - Corujeira, 1 km N, river banks (600 m), 11.ix.2003, 1 స̂ (all J. Roháček leg.).

Spain: Canary Is: Tenerife: Puerto de la Cruz [28²5'N 16³3'W], 8.ii.2014, 2 ${ }^{\circ}$ (M. Vála leg.).

Distribution: Only known from Portugal (Azores, Madeira) and Spain (Canary Is).

\section{Dicranomyia (Glochina) pauli Geiger, 1983}

Material examined:

Spain: Andalucía: Granada, Sierra Nevada, 3 km SW Berchules, Berchules de Lobras, shrubby canal (1300 m), 1.x.1989, 4 ふૈ, 1 क, 4.5 km SE Güéjar Sierra, Convento Alhambra, bank of brook, steep wooded ravine (1500 m), 28.ix.1989, 1 §̋; Jaén, Sierra de Cazoria, 8 km SW Bianquillo (Mt), Emb. Aguanderondo, clear forest with undergrowth, bank of brook (1000 m), 29.ix.1989, 1 §̃, 1 q (all C. Dufour \& J.-P. Haenni leg.).

Distribution: Great Britain, Italy, Macedonia, Switzerland; Israel. First records for Spain. 


\section{Dicranomyia (Glochina) sericata (Meigen, 1818)}

Material examined:

Portugal: Algarve: Tavira env., Currais Boieiros, river (27 m), 31.iii.2009, 2 o, 2 \%; Azinhal env., Barreiros, 1 km S, Beliche river (75 m), 2.iv.2009, 3 đ, 4.iv.2009, 1 § (all J. Starý leg.).

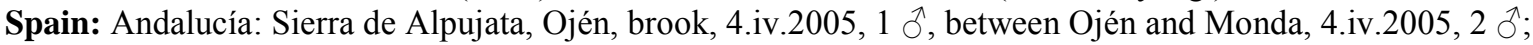

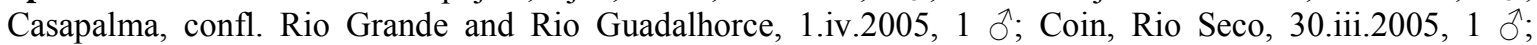
Alhaurin el Grande, 5 km E, 30.iii.2005, 1 ô, 1 क ; Alhaurin de la Torre, 31.iii.2005, 3 ô (all J. Starý leg.); Benalmádena, 29.iii.2005, 1 §̂, 6.iv.2005, 1 क (M. Vála leg.).

Distribution: Europe (unrecorded from Portugal), except for northern countries; Morocco; Azerbaijan, Turkey. First records for Portugal.

\section{Dicranomyia (Idiopyga) halterella Edwards, 1921}

Material examined:

Spain: Cataluña: Pyrenees Mts, Val de Núria, along brook (cca 2000 m), sweeping, 9.x.2008, 1 ô (J. Ševčík leg.).

Distribution: Nearctic (USA); widespread over Palaearctic (unrecorded from Portugal and Spain), including Central Asia and Mongolia, as far east as Far East of Russia. First record for Spain.

\section{Dicranomyia (Melanolimonia) hamata Becker, 1908}

Material examined:

Portugal: Algarve: Serra de Monchique, Alcaria do Peso, brooks (450-500 m), 3.iv.2009, 2 ô, 1 ค; Tavira

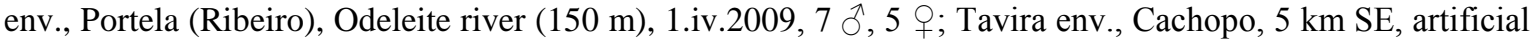
lake (210 m), 1.iv.2009, 1 ㅇ (all J. Starý leg.).

Spain: Andalucía: Alhaurin el Grande, $5 \mathrm{~km} \mathrm{E}$, 30.iii.2005, 2 ㄱ; Alhaurin de la Torre, 31.iii.2005, 8 ô, 1 우 (all J. Starý leg.). Canary Is: Tenerife: La Esperanza, $2 \mathrm{~km} \mathrm{SW}$, sweeping undergrowth of pine forest, 2.v.1999, 3 đ; Aguamansa env., sweeping vegetation along brook, 12.v.1999, 1 đ (all J. Roháček leg.).

Distribution: France (Corsica), Portugal (Azores), Spain (Canary Is); Turkey. First records for continental Portugal and continental Spain.

\section{Dicranomyia (Melanolimonia) morio (Fabricius, 1787)}

Material examined:

Portugal: Algarve: Serra de Monchique, Caldas de Monchique, brooks (230 m), 3.iv.2009, 1 ○ (J. Roháček

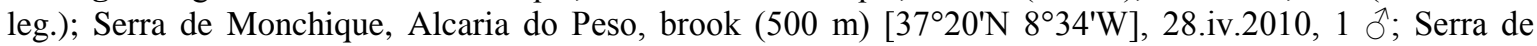

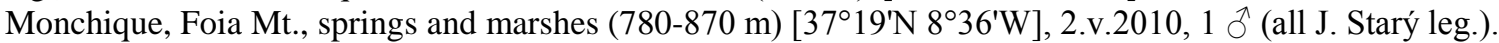

Distribution: Europe (unrecorded from Portugal); Morocco; Transcaucasia, Turkey, Iran; Mongolia. First records for Portugal.

\section{Dicranoptycha fuscescens (Schummel, 1829)}

Material examined:

Portugal: Setúbal: Serra Arrabida, Vila Nogueira, 30.v.1998, 1 ô (A. Čelechovský leg.). Algarve:

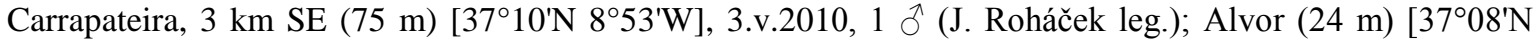
$8^{\circ} 35^{\prime} \mathrm{W}$ ], 26.iv.2010, 1 ○े (M. Vála leg.); Rasmalho, $2.5 \mathrm{~km} \mathrm{~N}$, Boina river $(75 \mathrm{~m})\left[37^{\circ} 15^{\prime} \mathrm{N} 8^{\circ} 33^{\prime} \mathrm{W}\right.$ ],

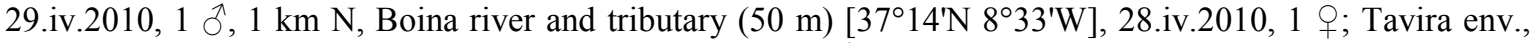
Portela (Ribeiro), Odeleite river $(150 \mathrm{~m}), 1 . i v .2009,2$; ; Tavira env., Currais Boieiros, river $(27 \mathrm{~m})$,

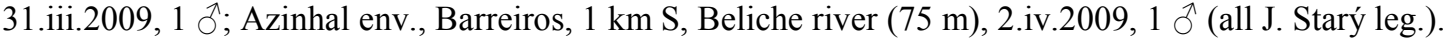

Distribution: Europe (unrecorded from Portugal); Morocco, Algeria; Transcaucasia, Turkey, Cyprus, Lebanon, Israel; ?Kazakhstan; Mongolia. First records for Portugal.

\section{Discobola freyana (Nielsen, 1961)}

Material examined:

Portugal: Azores: São Miguel I.: Caldeira Velha (Caldeiras $2 \mathrm{~km} \mathrm{SW}$ ), leafy forest, 7.ix.2006, 6 ô, 1 \%; Sete Cidades, Lagoa Verde, mixed forest, 8.ix.2006, 4 ô (all J. Roháček leg.); Santo Anttonio, Da Atalhada reserve, wet leafy forest, 6.ix.2006, $1 \hat{\jmath}, 1$; ; Caldeiras nr. Ribeira Grande, spring places, 5.ix.2006, $1 \hat{\jmath}$ (all J. Roháček \& M. Vála leg.).

Distribution: Only known from Portugal (Azores). 


\section{Geranomyia bivittata Becker, 1908}

Material examined:

Portugal: Madeira: Ponta Delgada [32 $\left.{ }^{\circ} 50^{\prime} \mathrm{N} 16^{\circ} 59^{\prime} \mathrm{W}\right]$, 4.iii.2011, 1 ㅇ (M. Vála leg.); Rabaçal env. (950

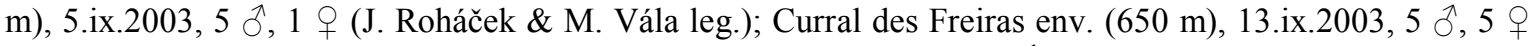
(J. Roháček leg.); Funchal - Corujeira, 1.5 km N (600 m), 13.ix.2003, 2 đ, 1 f (all M. Vála leg.).

Distribution: Only known from Portugal (Madeira).

\section{Helius (Helius) calviensis Edwards, 1928}

Material examined:

Portugal: Algarve: Serra de Monchique, Alcaria do Peso, brook (500 m) [37 $\left.20^{\prime} \mathrm{N} 8^{\circ} 34^{\prime} \mathrm{W}\right], 28 . i v .2010,1 \delta^{\wedge}$; Tavira, $3 \mathrm{~km} \mathrm{~N}$, Rio Ségua valley, drainages (24 m), 1.iv.2009, 1 +; Azinhal env., Barreiros, 1 km S, Beliche river (75 m), 2.iv.2009, 1 §̂, 2 q (all J. Starý leg.).

Spain: Andalucía: Fuengirola, Rio de Feungirola, 29.iii.2005, 1 đ (J. Starý leg.); Benalmádena, 6.iv.2005, 1 đ (M. Vála leg.), 9.IV.2005, 1 \& (J. Starý leg.). Región de Murcia: Lorca, near river (820 m), 19.viii.2006, 1 đ (M. Barták leg.). Balearic Is: Mallorca: S'Albufera National Park, "Cami des Polls", saltmarsh and canal

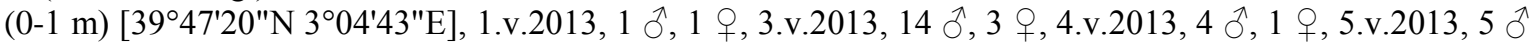
(J. Starý leg.).

Distribution: Bulgaria, France (Corsica), Italy (incl. Sardinia, Sicily), Spain (incl. Balearic Is: Ibiza); Algeria, Tunisia; Turkey. First records for Portugal and Spain (Balearic Is: Mallorca).

\section{Helius (Helius) hispanicus Lackschewitz, 1928}

Material examined:

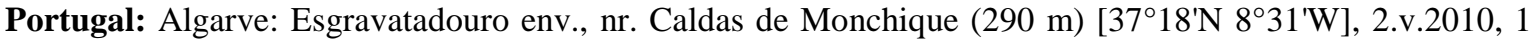
$\widehat{\jmath}, 1$ q (J. Starý leg.).

Spain: Andalucía: Sierra Palmitera, nr. Puerto del Madroño, 8.iv.2005, 1 \& (J. Starý leg.); Granada, Sierra Nevada, W of Lanjarón, thermal springs, riverside forest (700 m), 1.x.1989, 1 ô (C. Dufour \& J.-P. Haenni leg.).

Distribution: Great Britain, Spain; Morocco; Georgia, Azerbaijan, Turkey, Cyprus, Iran. First record for Portugal.

\section{Helius (Helius) pallirostris Edwards, 1921}

Material examined:

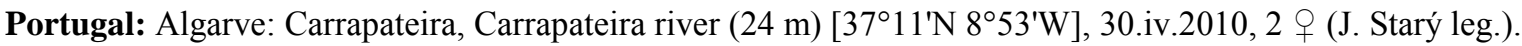
Spain: Balearic Is: Mallorca: S'Albufera National Park, "Cami de ses Puntes", saltmarsh $(0-1 \mathrm{~m})$ [3946'57"N 307'01"E], 1.v.2013, 1 § (J. Starý leg.).

Distribution: Europe (unrecorded from Portugal and Spain); Tunisia; Azerbaijan, Israel, Iran; Central Asia. First records for Portugal and Spain (Balearic Is).

\section{Limonia hercegovinae (Strobl, 1898)}

Material examined:

Portugal: Algarve: Serra de Monchique, Alcaria do Peso, brooks (450-500 m), 3.iv.2009, 2 § , brook (500

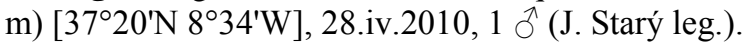

Spain: Andalucía: Sierra Palmitera nr. Puerto del Madroño, 5.iv.2005, 1 ㅇ (J. Starý leg.).

Distribution: Europe (unrecorded from Portugal), except for northern countries; Morocco; Azerbaijan, Turkey, Iran. First records for Portugal.

\section{Limonia nubeculosa Meigen, 1804}

Material examined:

Portugal: Algarve: Azinhal env., Barreiros, 1 km S, Beliche river (75 m), 2.iv.2009, 1 \& (J. Starý leg.).

Spain: Andalucía: Cortijo Los Pinos (nr. Alozaina), road bridge, 3.iv.2005, 1 +; Alhaurin el Grande, 5 km E, 30.iii.2005, 1 ㅇ (all J. Starý leg.). Balearic Is: Mallorca: Sant Miquel nr. Campanet, Ses Fonts Utanes

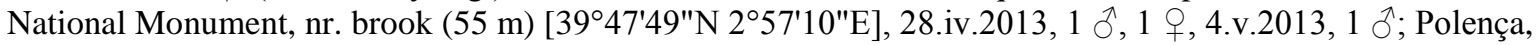

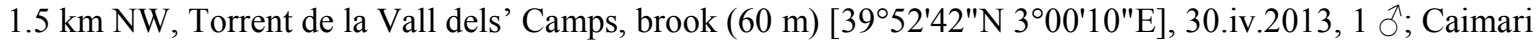
nr. Selva, $0.6 \mathrm{~km} \mathrm{NW}$, ravine (210 m) [3946'30"N 2 53'52"E], 5.v.2013, 1 ㅇ (all J. Starý leg.).

Distribution: Nearctic (Canada, USA); widespread over Palaearctic, including North Africa, Central Asia, and Mongolia, as far east as Far East of Russia, North Korea, and Japan. 


\section{Neolimonia dumetorum (Meigen, 1804)}

\section{Material examined:}

Portugal: Algarve: Serra de Monchique, Foia Mt., sprins and marshes $(780-870 \mathrm{~m})\left[37^{\circ} 19^{\prime} \mathrm{N} 8^{\circ} 36^{\prime} \mathrm{W}\right]$,

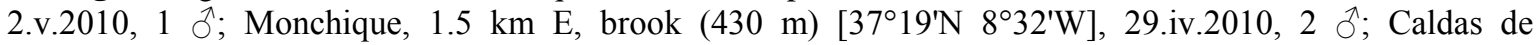
Monchique, spring ( $230 \mathrm{~m})\left[37^{\circ} 17^{\prime} \mathrm{N} 8^{\circ} 33^{\prime} \mathrm{W}\right], 28 . i v .2010,1$ ㅇ (all J. Starý leg.).

Distribution: Europe (unrecorded from Portugal); Georgia, Azerbaijan. First records for Portugal.

\section{Thaumastoptera (Thaumastoptera) insignis Lackschewitz, 1940}

\section{Material examined:}

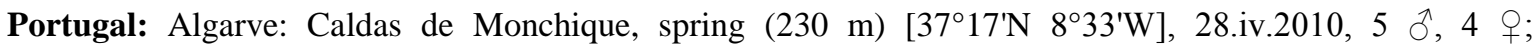

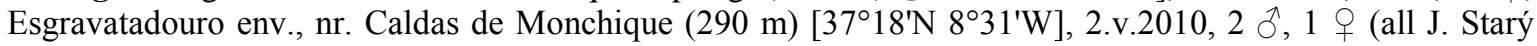
leg.).

Distribution: Only known from Spain. First records since original description; first records for Portugal.

\section{PEDICIIDAE}

\section{Dicranota (Paradicranota) pallens Lackschewitz, 1940}

Material examined:

Spain: Andalucía: Sierra Palmitera nr. Puerto del Madroño, 5.iv.2005, 1 $\widehat{\jmath}$, 8.iv.2005, $3 \hat{\jmath}$ (J. Starý leg.).

Distribution: Albania, Austria, Bulgaria, Czech Republic, Germany, Italy, Poland, Romania, Slovakia, Spain, Switzerland; North Caucasus.

\section{Tricyphona (Tricyphona) immaculata (Meigen, 1804)}

\section{Material examined:}

Portugal: Algarve: Serra de Monchique, Alcaria do Peso, brooks (450-500 m), 3.iv.2009, 1 §, Foia Mt.,

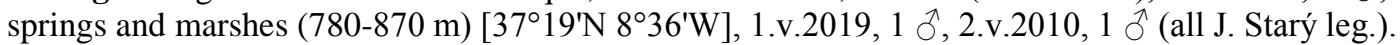

Distribution: Europe (unrecorded from Portugal); Transcaucasia, Turkey, Lebanon; West Siberia; Central Asia. First records for Portugal.

\section{Results and discussion}

This paper presents additions to the fauna of the families Limoniidae and Pediciidae for the territories of Portugal and Spain. With these additions numbers of species known from the individual territorial units are given below (Limoniidae and Pediciidae are separated by a slash), followed, in parentheses, by previous numbers based on Oosterbroek (2014): Portugal (continental) - 47/3 (16/2); Azores - 9/0 (9/0); Madeira - 14/0 (12/0); Spain (continental) 122/9 (117/9); Balearic Islands - 18/0 (6/0); Canary Islands 27/0 (25/0). A considerable increase in the number of species for the continental Portugal is caused by previous little knowledge of this fauna. The same is true for the Balearic Islands. Portugal is still insufficiently explored and deserves more rigorous investigation than any other area of the territory. Nevertheless, four species, so far unknown from Spain, viz. Euphylidorea aperta, Achyrolimonia decemmaculata, Dicranomyia (D.) patricia, and Helius (H.) pallirostris, are here newly recorded for Portugal. Occurrence of Molophilus (M.) griseus is here confirmed for the continental Spain. Previous records of Dicranomyia (D.) ornata from both Portugal and Spain should be checked for whether these do not refer to $D$. (D.) patricia, a species very similar to D. (D.) ornata in its conspicuous wing pattern. Since the Macaronesian archipelagos are probably the best-known areas (as to the Limoniidae), also because of their faunas comparatively poor in species, even though significantly endemic, faunal novelties from there would be of interest: Austrolimnophila (A.) analis and Atypophthalmus (A.) quinquevittatus are here newly recorded from Madeira, and Dicranomyia (D.) maderensis and $D$. (D.) modesta from the Canary Islands. The latter species seems to be a recent immigrant from Europe. Four species are here recorded for the first time since their original descriptions, 
viz. Baeoura ebenina, Scleroprocta oosterbroeki, Dicranomyia (D.) patricia, and Thaumastoptera (T.) insignis.

Acknowledgements: First of all, I thank my companions on collecting trips, J. Roháček (Slezské zemské museum, Opava, Czech Republic) and M. Vála (Olomouc, Czech Republic), for their assistance in the field and collections of craneflies made during their Macaronesian trips, in which I was not a participant. Other colleagues, viz. M. Barták (Česká zemědělská univerzita, Praha, Czech Republic), A. Čelechovský (Univerzita Palackého, Olomouc, Czech Republic), C. Dufour and J.-P. Haenni (Muséum d’histoire naturelle, Neuchâtel, Switzerland), P. Oosterbroek and C. Hartveld (Amsterdam, the Netherlands), and J. Ševčík (Ostravská univerzita, Ostrava, Czech Republic), contributed with individual specimens. The paper was financially supported by the Ministry of Culture of the Czech Republic by institutional financing of long-term conceptual development of the research institution (the Silesian Museum, MK000100595), internal grant of the Silesian Museum No. IGS201303/2013.

\section{References}

Báez M. \& García A. (2004): Limoniidae, pp. 270-271. In Izquierdo I., Martín J.L., Zurita N. \& Arechavaleta M. (eds): Lista de especies silvestres de Canarias (hongos, plates y animales terrestres). Consejería de Medio Ambiente y Ordenación Territorial, Gobierno de Canarias, 499 pp. (in Spanish).

Carles-Tolrá M., Calvo F. \& Zabalegui I. (2006): Nuevos datos sobre dípteros capturados en el Parque Natural de Aiako Harria (España, País Vasco: Gipuzkoa) (Insecta: Diptera). - Heteropterus Revista de Entomología 6: 95-103. (in Spanish).

Carles-Tolrá M. \& Lencina J.L. (2010): Algunos dípteros capturados en la Región de Murcia y otras provincias españolas, mediante trampas de interceptación de vuelo (España) (Insecta, Diptera). - Boletín de la Sociedad Entomológica Aragonesa 46: 483-489. (in Spanish).

Carles-Tolrá M. \& Ventura D. (2009): Dípteros nuevos para las Islas Baleares (Insecta: Diptera). Heteropterus Revista de Entomología 9: 161-163. (in Spanish).

Eiroa E. (2008): Limoniidae, p. 333. In Borges P.A.V., Abreu C., Aguiar A.M.F., Carvalho P., Jardim R., Melo I., Oliveira P., Sérgio C., Serrano A.R.M. \& Vieira P. (eds): Listagem dos fungos, flora e fauna terrestres dos arquipélagos da Madeira e Selvagens. A list of the terrestrial fungi, flora and fauna of Madeira and Selvagens archipelagos. 440 pp. Direcção Regional do Ambiente da Madeira and Universidade dos Açores, Funchal and Angra do Heroísmo, 440 pp. (in Portuguese and English).

Eiroa E. \& Báez M. (2002): Limoniidae, pp. 54-57. In Carles-Tolrá M. (ed.): Catálogo de los Diptera de España, Portugal y Andorra (Insecta). Monografía Sociedad Entomologica Aragonesa, vol. 8, Zaragoza, 323 pp. (in Spanish, Portuguese, and English).

Oosterbroek P. (2014): Catalogue of the Craneflies of the World (Insecta, Diptera, Nematocera, Tipuloidea). Version 21 March 2014 [online]. Available from http://ip30.eti.uva.nl/ccw/

Starý J. (2006a): New information resulting from a study of Strobl’s types of Limoniidae (Diptera). - Acta Universitatis Carolinae, Biologica 49 (2005): 187-203.

- (2006b): Hoplolabis (Parilisia) species related to H. (P.) punctigera (Lackschewitz, 1940) and H. (P.) spinosa (Nielsen, 1953) with the description of a new species (Diptera, Limoniidae). - Studia dipterologica 13: 115125.

- (2008): Three new European species of the genus Scleroprocta Edwards, 1938 (Diptera: Limoniidae). Biologia, Bratislava 63: 120-126.

- (2009a): West Palaearctic species of the genus Eloeophila (Diptera: Limoniidae). - European Journal of Entomology 106: 425-440.

- (2009b): The identity of Dicranomyia (Dicranomyia) luteipennis Goetghebuer (Diptera, Limioniidae). Zootaxa, 2155: 55-68.

- (2011): Descriptions and records of the Palaearctic Molophilus Curtis (Diptera, Limoniidae). - Zootaxa 2999: 45-62.

- (2012): The identity of Geranomyia bezzii and a description of G. fuscior sp. nov. (Diptera: Limoniidae). Acta Entomologica Musei Nationalis Pragae 52: 273-279.

Starý J. \& Oosterbroek P. (2008): New records of West Palaearctic Limoniidae, Pediciidae and Cylindrotomidae (Diptera) from the collections of the Zoological Museum, Amsterdam. - Zootaxa 1922: 120.

Vieira V., Diaz S. \& Báez M. (2010): Limoniidae, p. 237. In Borges P.A.V., Costa A., Cunha R., Gabriel R., Gonçalves V., Martins A.F., Melo I., Parente M., Raposeiro P., Rodrigues P., Santos R.S., Silva L., Vieira P. \& Vieira V. (eds): Listagem dos organismos terrestres e marinhos dos Açores. A list of the terrestrial and marine biota from the Azores. Princípia, Cascais, 432 pp. (in Portuguese and English). 
Author's address: Jaroslav S t a r ý, Neklanova 7, CZ-779 00 Olomouc-Nedvězí \& Silesian Museum, Tyršova 1, CZ-746 01 Opava, Czech Republic; e-mail: stary.cranefly@gmail.com 